\title{
Selective excision of adenomas originating in or extending into the pituitary stalk with preservation of pituitary function
}

\author{
R. Bryan Mason, M.D., Lynnette K. Nieman, M.D., John L. Doppman, M.D., and Edward H. \\ Oldfield, M.D.
}

Surgical Neurology Branch, National Institute of Neurological Disorders and Stroke; Developmental Endocrinology Branch, National Institute of Child Health and Human Development; and Diagnostic Radiology Department, The Clinical Center, National Institutes of Health, Bethesda, Maryland; and Department of Neurosurgery, National Naval Medical Center, Bethesda, Maryland

When the surgeon identifies an adenoma within the gland and selectively excises it, endocrine-active pituitary tumors are usually cured and pituitary function is preserved. Occasionally ectopic adenomas arise primarily in the pituitary stalk or arise superiorly in the midportion of the anterior lobe and extend upward within the stalk. To determine if these tumors can be selectively excised with preservation of pituitary function, the authors examined the outcome of selective adenomectomy in 10 patients with Cushing's disease with an ectopic adenoma originating in, and confined to, the stalk (four microadenomas) or an adenoma extending superiorly through the diaphragma sella and into the stalk (five microadenomas and one macroadenoma) from an operative series of 516 patients with Cushing's disease.

To reach the adenoma transsphenoidally in these patients, the diaphragma sella was incised anteroposteriorly in the midline to the anterior edge of the stalk, the suprasellar cistern was entered, and the adenoma was selectively excised using care to limit injury to the infundibulum. After selective adenomectomy, Cushing's disease remitted in all patients. All patients were hypocortisolemic immediately after surgery and required hydrocortisone for up to 21 months. Apart from the adrenal axis, pituitary function was normal in five patients in the immediate postoperative period, including two patients with tumors confined to the stalk and three patients with preoperative hypothyroidism (one patient) and/or hypogonadism (three patients). Three others with transient postoperative hypothyroidism (one patient) or diabetes insipidus (two patients) had normal pituitary function within 7 months of surgery. One patient with hypothyroidism and one with hypogonadism before surgery had panhypopituitarism postoperatively. A patient with a microadenoma located high in the stalk next to the optic chiasm had bitemporal hemianopsia postoperatively.

These results demonstrate the feasibility of achieving curative transsphenoidal resection and preservation of pituitary function in cases of pituitary adenomas that are confined to the pituitary stalk or tumors that extend superiorly within the stalk from an intrasellar origin.

Key Words * pituitary adenoma * Cushing's disease * pituitary stalk 
Most pituitary adenomas arise in the anterior lobe of the pituitary gland and can be excised selectively via transsphenoidal adenomectomy with elimination of tumor and preservation of pituitary function. However, some adenomas arise within, and remain localized to, the pituitary stalk. Other pituitary adenomas arise in the superior portion of the anterior lobe and extend superiorly into the stalk. In the past, suprasellar ectopic pituitary adenomas that involve the stalk have been removed by transcranial surgery.[3,6,12,16,19,21,24,26] Using these operations, tumor removal was often incomplete and most patients lost pituitary function.[6,16,24,26] We report 10 cases of patients with adrenocorticotropic hormone (ACTH)-secreting pituitary adenomas that originated in the infundibulum or extended into the infundibulum through the opening of the diaphragma sella. All patients underwent transsphenoidal selective adenomectomy and showed laboratory and clinical remission of their hypercortisolism. Seven patients with normal pituitary function before surgery retained normal function after surgery, one patient with abnormal pituitary function before surgery regained normal function, and two patients with impaired pituitary function preoperatively lost additional pituitary function as a result of the surgery.

\section{CLINICAL MATERIALS AND METHODS}

\section{Patient Population}

Ten patients with adenomas that arose in the pituitary stalk above the diaphragma sella or extended superiorly into the stalk and above the diaphragma sella from the anterior lobe were identified from among 516 consecutively admitted patients who underwent transsphenoidal exploration for Cushing's disease at the National Institutes of Health between 1982 and 1995. Six patients (two women and four men, 26-60 years old) had adenomas that extended superiorly from the anterior pituitary into the stalk. One of these tumors was a macroadenoma $(>=10-\mathrm{mm}$ maximum diameter). Four patients (two male and two female patients, 14-34 years old) had microadenomas that originated within, and remained confined to, the pituitary stalk.

All 10 patients underwent a standard magnetic resonance (MR) imaging protocol[11] performed using a 1.5-tesla imaging system (Signa; General Electric, Minneapolis, MI). Studies included $\mathrm{T}_{1}$-weighted coronal and sagittal images of the pituitary fossa in 3-mm sections. Gadolinium-diethylenetriamine pentaacetic acid (DTPA) (0.1 mmol/kg body weight [Magnevist; Berlex Lab, Wayne, NJ]) was administered intravenously over 2 minutes. The $\mathrm{T}_{1}$-weighted coronal images were then repeated. All surgical procedures were performed by the same surgeon (E.H.O.).

\section{Laboratory Evaluation}

Preoperative determination of hypercortisolism and diagnosis of Cushing's disease were established using current conventional endocrinological and radiological evaluation. The biochemical response to surgery was measured according to a standardized protocol. Each patient received $0.5 \mathrm{mg}$ of dexamethasone intravenously every 6 hours for 36 hours beginning immediately after surgery. On the 3 rd postoperative day ( $>=24$ hours after the last $0.5-\mathrm{mg}$ dose of dexamethasone), morning serum cortisol levels were drawn between 7 a.m. and 8 a.m., and urine was collected for a 24-hour urinary free cortisol (UFC) test. These determinates were repeated for several consecutive days. A response to surgery demonstrating remission of hypercortisolism was classified as hypocortisolism (morning cortisol level < $7 \mu \mathrm{g} / \mathrm{dl} ; \mathrm{UFC}<20 \mu \mathrm{g} /$ day) or eucortisolism (UFC 20-90 $\mu \mathrm{g} /$ day).

Pituitary hypothyroidism was defined as subnormal levels of free tyroxin $(<0.9 \mathrm{mg} / \mathrm{dl})$ with low or subnormal thyroid-stimulating hormone values. Hypogonadism was defined as the absence of menses in 
a woman of reproductive age, inappropriately low follicle-stimulating hormone $(<25 \mathrm{ng} / \mathrm{ml})$ in an amenorrheic woman older than 50 years of age or subnormal plasma testosterone $(<200 \mathrm{ng} / \mathrm{ml})$ in a man. Partial hypopituitarism was defined as a deficiency in one or more, but not all, pituitary hormones. Panhypopituitarism was defined as a deficiency in all anterior pituitary hormones. Hypopituitarism was considered a result of surgery if hormonal replacement that was not needed preoperatively became necessary postoperatively. The immediate postoperative period was designated as the time from surgery to 3 months afterward, when the late postoperative period began.[27]

\section{Surgical Technique}

In all patients a transsphenoidal approach was used to resect the adenoma selectively (Fig. 1). Adenomas in the stalk always reached the pituitary capsule or the pia-arachnoid and, thus, lacked the type of capsule seen in adenomas of the pituitary gland. In contrast to intrasellar adenomas, which generally are quite easy to separate from surrounding normal tissue, the margin of adenomas within the stalk was more adherent to the stalk than is characteristic of the interface of the pseudocapsule of an adenoma and the anterior lobe. To develop a clear delineation of the pseudocapsule surrounding the adenoma and to separate the edge of the tumor from the stalk, an incision along the interface between the pseudocapsule and the stalk was made and developed with a No. 11 or No. 15 scalpel on a long bayonet handle. Blunt dissection using fine bipolar tips, microdiscs, and small ring curettes was performed to define the margin of the adenoma from the pituitary gland and to remove the remaining tumor. 


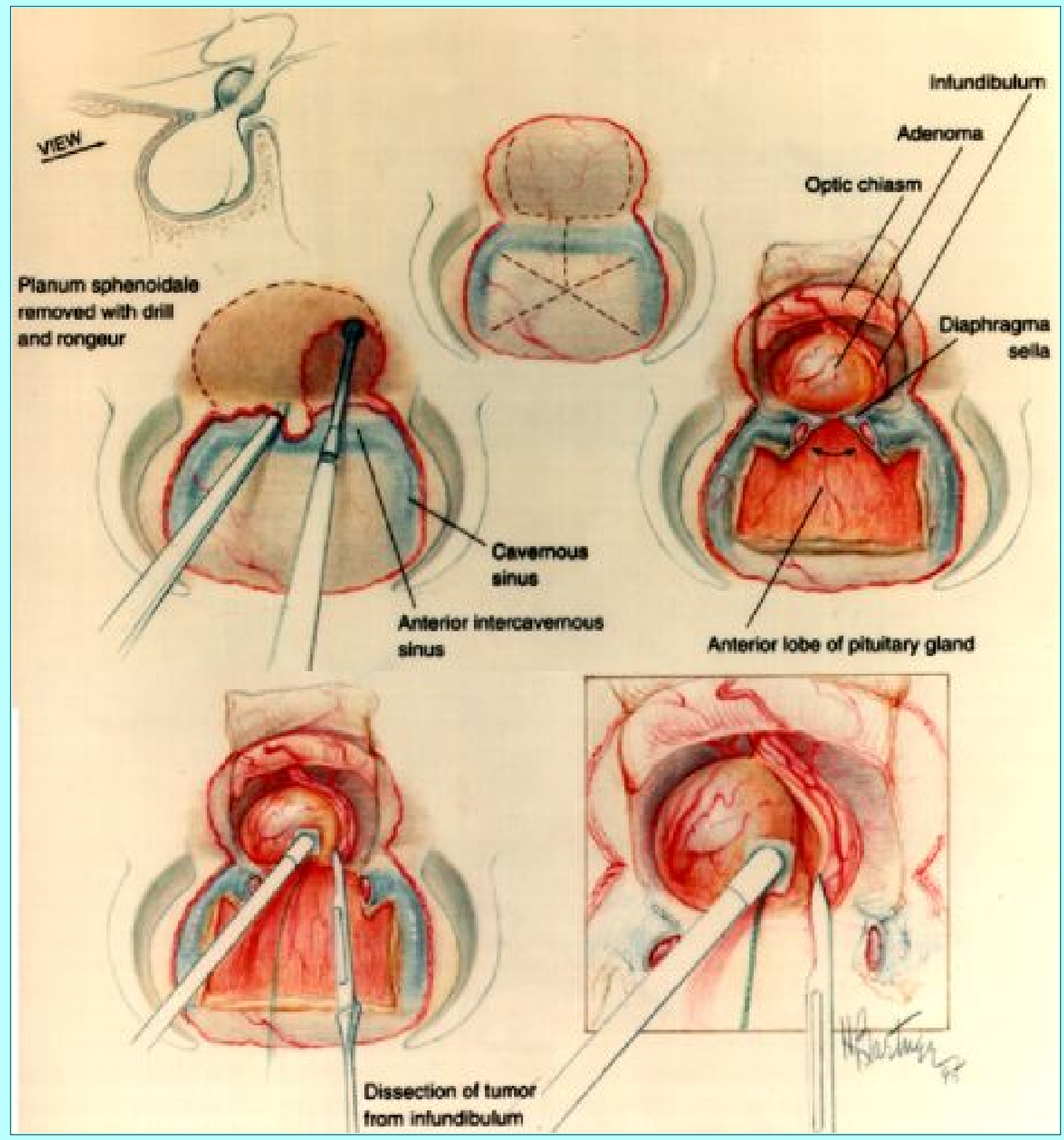

Fig. 1. Drawings depicting the routine wide exposure of the anterior surface of the sella and bone removal to expose the medial portion of the cavernous sinus bilaterally. In patients in whom MR imaging indicated stalk involvement, the posterior portion of the planum sphenoidale (the posterior 4-6 $\mathrm{mm}$ ) was removed by first drilling with a rough diamond burr until the plate of bone was paper thin, and then using a 2-mm-thin-footplate cervical Kerrison rongeur. Removal of the planum sphenoidale aided access to the suprasellar cistern, the pituitary stalk, and the superior surface of the gland. The dura mater covering the anterior pituitary surface was opened widely. Bilateral parasagittal incisions were made 8 to $10 \mathrm{~mm}$ apart in the dura overlying the planum sphenoidale. A transverse incision just above the anterior portion of the circular sinus was then used to connect the parasagittal incisions. The resulting dural flap was opened posteriorly while, initially, preserving the intact arachnoid. After entering the suprasellar cistern, the superior hypophyseal artery was identified and care was taken not to injure it. The exposed diaphragma sella was incised in the midline, in an anteroposterior direction to reach the stalk and the supradiaphragmatic 
tumor. A small piece of Gelfoam or cottonoid was placed superiorly in the subarachnoid space to prevent passage of blood into the CSF. Characteristic vertical striations produced by the vertical course of the surface blood vessels permitted identification of the stalk. A sharp incision was made in the pia-arachnoid with a No. 11 or a No. 15 scalpel at the junction of the capsule of the tumor with the pituitary stalk and, when appropriate, in the superior surface of the anterior lobe at the margin of the adenoma. The adenoma was then resected using standard microsurgical techniques.

\section{RESULTS}

\section{Magnetic Resonance Imaging and Surgery}

In three of the six patients with tumor arising in the anterior lobe and extending into the stalk (Fig. 2), MR imaging delineated the locus of the adenoma within the sella and demonstrated the supradiaphragmatic extension (Cases 2, 3, and 4; Table 1).

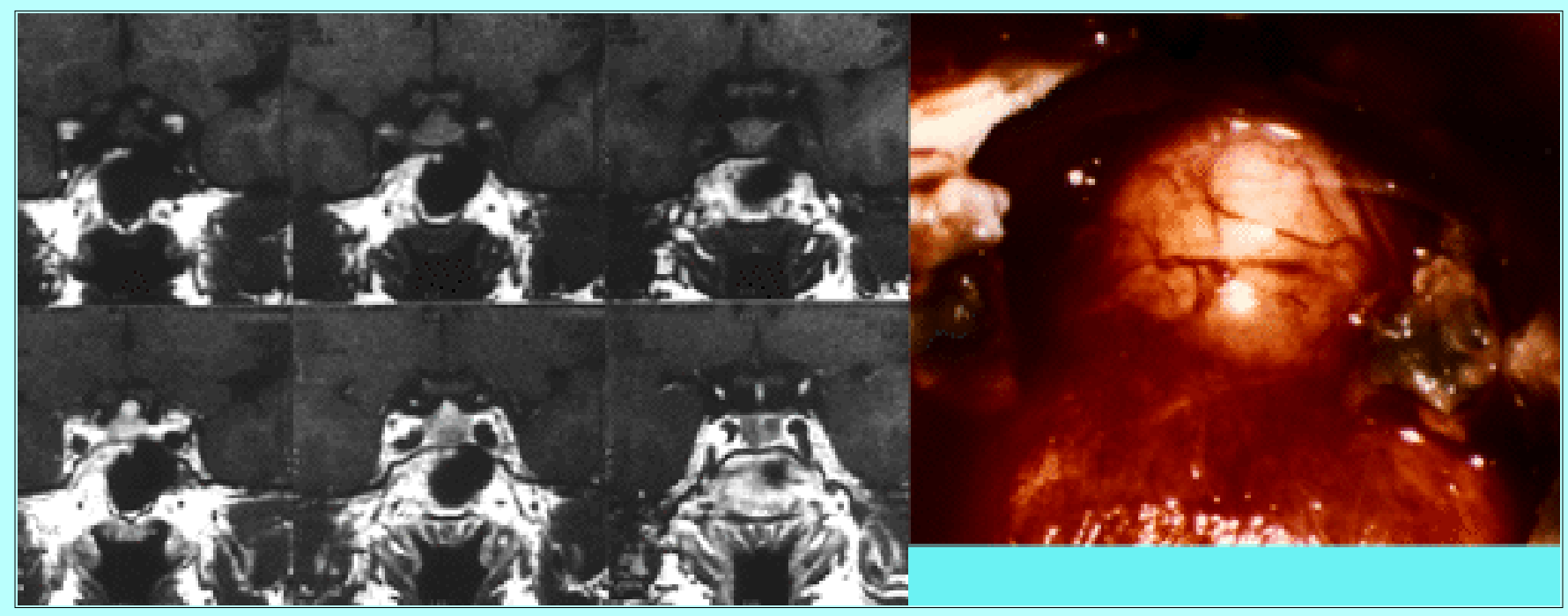

Fig. 2. Case 3. Upper: Magnetic resonance $T_{1}$-weighted images showing an adenoma on the superior surface of the gland extending upward through the diaphragma sella into the pituitary stalk. The images in the top row were obtained before contrast enhancement with gadolinium DTPA. Lower: Intraoperative photograph showing the adenoma (spherical, yellow-white mass) on the superior surface of the gland extending into the stalk. The coagulated tissues on each side are the anterosuperior intercavernous sinuses at the level of the diaphragma sella.

In the patient in Case 5, in whom the tumor arose between the anterior and posterior lobes and extended through the diaphragma sella, the MR image was normal. In Case 1 the MR image did not correspond to the operative findings (the MR image showed an enhancing area on the right side of the gland but did not demonstrate the adenoma or the existing tumor invasion of the diaphragma sella). In the sixth patient (Case 6) there was a small area of extension above the diaphragma sella on the MR image that was not appreciated prospectively, but was apparent when the operative findings were known.

In three of the four patients with tumor confined to the stalk (Figs. 3 and 4), the MR image accurately demonstrated the tumor and its relationship with the stalk (Cases 7, 9, and 10). In one patient, the MR image revealed a signal void in the left half of the gland, but no tumor was found there at surgery and the tumor was entirely supradiaphragmatic. Thus, in six of the 10 patients the results of preoperative MR 
imaging corresponded with the intraoperative findings.
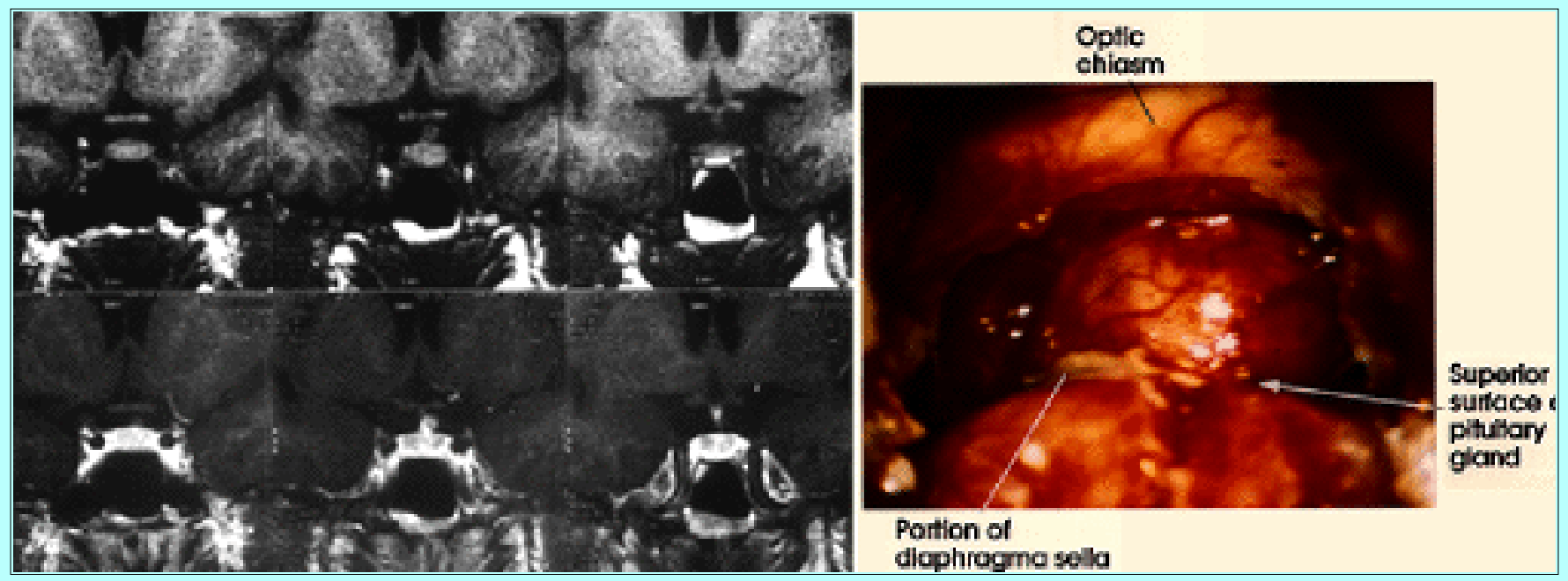

Fig. 3. Case 9. Upper: Magnetic resonance $\mathrm{T}_{1}$-weighted images showing an adenoma arising from the pituitary stalk. The images in the top row were obtained before contrast enhancement with gadolinium-DTPA. Lower: Intraoperative photograph of a pituitary adenoma confined exclusively to the stalk. The vertical incisions in the anterior lobe were used to explore the gland. Note the relationship of the superior portion of the tumor to the optic chiasm and the narrow remnant of the diaphragma sella between the superior surface of the pituitary and the adenoma.

In two patients with adenomas arising from the intrasellar portion of the anterior lobe, the arachnoid was preserved during dissection of the supradiaphragmatic portion of the tumor. The arachnoid of the suprasellar cistern was intentionally entered in the other eight patients. To remove cerebrospinal fluid (CSF) for exposure of the tumor and the contiguous infundibulum, positive pressure ventilation was used to deliver CSF into the field, where it was aspirated. After tumor removal,the opening in the arachnoid and diaphragma sella was occluded by an abdominal fat graft in the eight patients who underwent arachnoid exploration. A lumbar CSF drain was placed at surgery in five patients while general anesthesia was still in effect. These patients remained at flat bedrest for 3 to 5 days of continuous CSF drainage. The five patients in whom CSF was not drained were kept flat in bed for 2 to 3 days. None of the patients had a persistent CSF leak after surgery. 


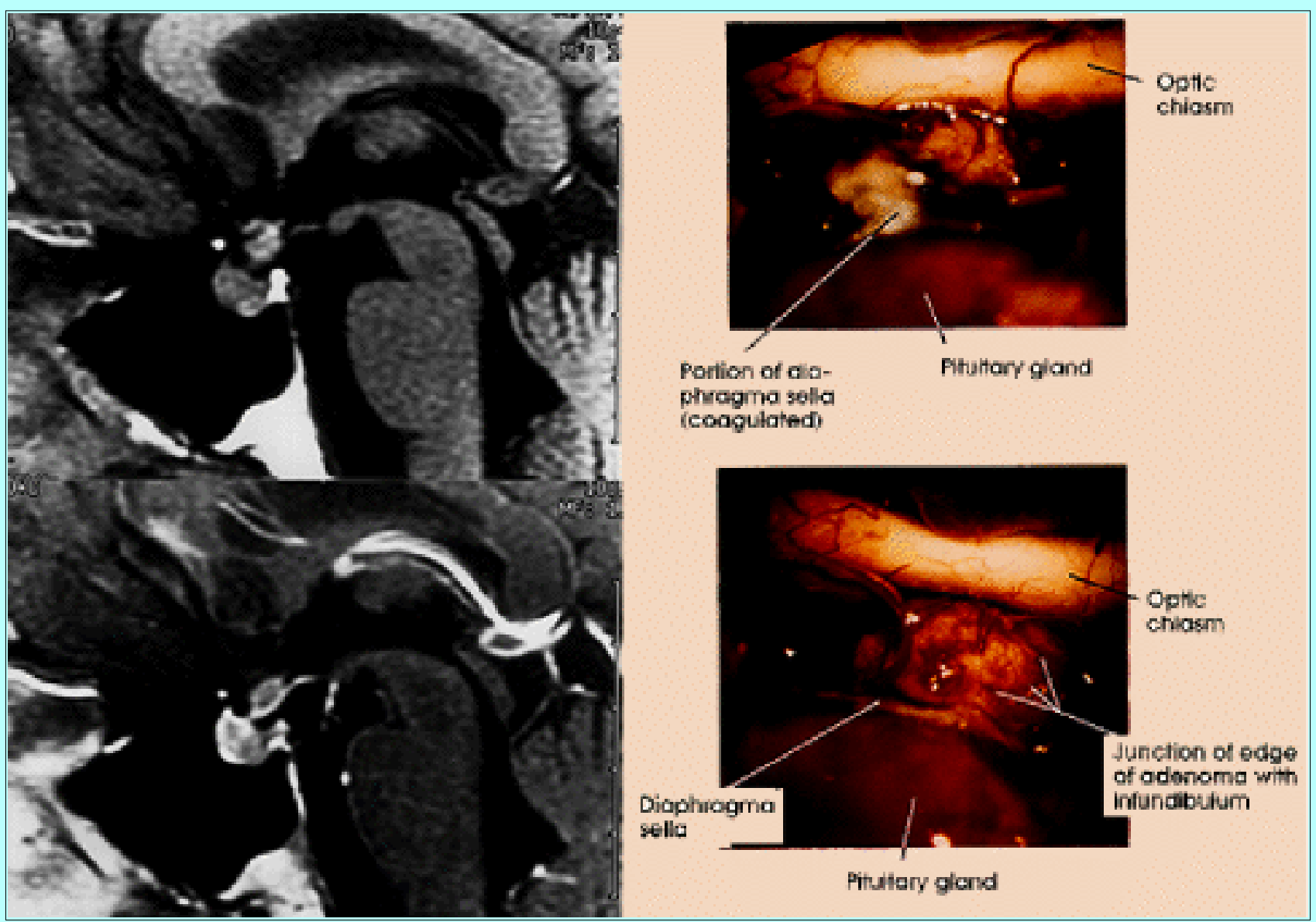

Fig. 4. Case 10. Upper and Lower Left: Magnetic resonance $T_{1}$-weighted images revealing a 7-mm adenoma arising high in the pituitary stalk, just beneath the optic chiasm (sagittal view before [upper left] and after [lower left] contrast enhancement with gadolinium DTPA. Note enhancement of the anterior lobe and stalk, but not the tumor). Upper and Lower Right: Intraoperative photographs of a pituitary adenoma confined to the pituitary stalk with its superior margin contiguous with the optic chiasm. Initial exposure of the tumor (upper right) revealed a gray-white mass that was not contained by the capsule of the stalk, resulting in a papillary, irregular surface of the anterior aspect of the tumor. Note the course of the small branches of the superior hypophyseal arteries through the anterolateral margin of the tumor to reach the inferior and anterior surface of the chiasm (upper and lower left), and the interface between the edge of the adenoma and the stalk (lower right), which was displaced posteriorly and to the left by the microadenoma (lower right; view after removal of a portion of the adenoma outside the capsule of the infundibulum).

\section{Endocrinological Function}

All patients had diagnostic central-to-peripheral ACTH concentration gradients between the inferior petrosal sinuses and the peripheral blood during the inferior petrosal sinus sampling (IPSS) test. All patients exhibited ACTH-staining tumors on immunohistochemical analysis. With the exception of the hypercortisolism associated with Cushing's disease, four patients, including one of the four patients in whom the adenoma was confined to the stalk, had normal pituitary function before surgery. One patient with tumor originating in the anterior lobe and extending into the stalk suffered from hypothyroidism (Case 1) and one patient had amenorrhea (Case 3). Another patient who had a macroadenoma that originated in the anterior lobe had hypothyroidism and hypogonadism (Case 2). Three of the four patients 
whose tumor originated in the stalk had impaired pituitary function before surgery. One had amenorrhea, one had amenorrhea and hypothyroidism, and one had hypogonadism before surgery. No patient exhibited posterior pituitary dysfunction before surgery. The patient who had undergone previous transsphenoidal surgery (Case 4) had normal pituitary function.

All patients became hypocortisolemic in the early postoperative period. Nine patients became hypocortisolemic immediately after surgery and required cortisol supplementation at discharge (Table 1). One patient was eucortisolemic immediately after surgery, but developed symptomatic profound hypocortisolism 1 month after surgery and required glucocorticoid supplementation for 10 months (Case 7).

The pituitary stalk was preserved intact in all patients, although in two it was somewhat attenuated. Three of the four patients with tumor confined to the pituitary stalk and five of the six patients with tumor originating in the gland and extending into the stalk had normal anterior pituitary function after surgery (Table 1). The patient with a macroadenoma and preoperative hypothyroidism and hypogonadism (Case 2) had laboratory evidence of recovery of pituitary function at 3- and 6-month reassessment and required no further medical therapy. Two patients with preoperative hypothyroidism (Case 1) and hypogonadism (Case 10) required replacement of all anterior pituitary hormones (except growth hormone and prolactin) after surgery. Six of the 10 patients had normal posterior pituitary function at discharge from the hospitalization. Three patients had transient postsurgical diabetes insipidus (two patients with tumor extending into the stalk from the pituitary required 1-desamino-8-D-arginine vasopressin (DDAVP) for 3 and 7 months and one patient with adenoma exclusively in the stalk had diabetes insipidus for 20 months). Postoperative delayed endocrinological assessment has not been completed in the remaining patient (Case 10), who underwent surgery only recently.

Assessment in the late postoperative period included measurement of 24-hour UFC, diurnal cortisol levels, and the ACTH stimulation test to evaluate return of function to the hypothalamic-pituitary-adrenal axis. During a follow-up period lasting up to 42 months, eight patients continued to show clinical and laboratory evidence of remission. One patient (Case 7), who had eucortisolism immediately after surgery but became symptomatic from profound hypocortisolism 1 month later, developed clinical and laboratory evidence of recurrent Cushing's disease 31 months after surgery. The other patient (Case 10), who had undergone surgery recently, was hypocortisolemic in the immediate postoperative period. All patients required supplemental glucocorticoid therapy for a variable interval (Table 1). Seven patients have had recovery of the hypothalamic-pituitary-adrenal axis. This recovery was demonstrated by results of the ACTH stimulation test and by successful discontinuation of the replacement glucocorticoid medications. Two patients were surgically treated within the past 10 months and the other patient has panhypopituitarism.

In Case 10, MR imaging revealed a 7-mm enhancing mass abutting the inferior and posterior surface of the optic chiasm (Fig. 4) and neuroophthalmological assessment revealed diminished acuity in the left eye and a slight visual field deficit in the inferior and lateral quadrant of that eye. At surgery a 6- to 8-mm gray-white adenoma was identified contiguous with the inferior surface of the optic chiasm, lying in front of the stalk, which had been displaced posteriorly and to the left by the microadenoma. Two small vessels, which appeared to be branches of the superior hypophyseal artery and which were imbedded in the anterolateral edge of the tumor on each side, continued to extend beyond the tumor to reach the inferior and anterior surfaces of the optic chiasm. These vessels were interrupted to achieve complete removal of the tumor. After surgery the patient awoke with a bitemporal hemianopsia that 
affected the inferior quadrants with the greatest severity. There was no change in his visual fields as of his 3-week postoperative assessment, the last examination for which complete results are available.

\section{DISCUSSION}

The transsphenoidal approach and craniotomy are the surgical approaches to the suprasellar region. One criterion that has been used to indicate safe and successful use of the transsphenoidal approach to remove tumors that arise or extend above the diaphragma sella is the presence of an enlarged sella.[17] We have demonstrated the efficacy of the transsphenoidal approach for adenomas involving the pituitary stalk in 10 patients with a normal size sella.

Five of the eight previously reported patients with pituitary adenomas involving the pituitary stalk had Cushing's disease. $[7,19,26]$ The other three had a chromophobic adenoma,[12] a prolactin-secreting adenoma,[6] and an adenoma of unspecified type.[24] Three of the five patients with Cushing's disease initially underwent unsuccessful transsphenoidal surgery.[6] In those three cases, no tumor was identified in the gland and the supradiaphragmatic area was not explored. One of those patients underwent a subsequent craniotomy with sectioning of the stalk and removal of the tumor. Although cured of Cushing's disease, that patient required total hormone replacement. One other patient underwent gamma knife radiation therapy and had persistent disease at the time of the report. The third patient had no further surgery and suffered from persistent disease. Two patients underwent craniotomy only: one was cured, but the stalk was sectioned and hormone replacement therapy was required,[26] and the other one died of postoperative complications.[16] In a more favorable report, a patient with a nonsecreting adenoma involving the stalk underwent a craniotomy with tumor removal and preservation of pituitary function.[12] Thus, because none of the five patients with Cushing's disease had curative transsphenoidal surgery with preservation of pituitary function, Dyer, et al.,[6] concluded: "The cranial approach remains the only operative approach to lesions in the supradiaphragmatic region." Although two of the three patients with craniotomy were cured of Cushing's disease, they both lost pituitary function; the third patient did not survive.

The success of transsphenoidal surgery is enhanced by accurate preoperative localization of the tumor. When the MR image is equivocal or normal, as was the case in four of our patients, the IPSS test may confirm the presence of sellar or ectopic pituitary tumor. With supradiaphragmatic tumors, it was not established previously whether the venous drainage reached the cavernous sinus and the inferior petrosal sinuses. In all four of these patients the results met the diagnostic criteria[20] for Cushing's disease during the IPSS test. If no tumor is identified preoperatively by MR imaging (as occurred in four of our 10 patients) and no adenoma is evident at pituitary exploration, despite endocrinological results that meet stringent criteria for a diagnosis of Cushing's disease, $[9,20]$ the physician should consider the possibility of an ectopic ACTH-secreting pituitary adenoma that arises in the cavernous sinus[28] or in the suprasellar cistern and should have the imaging studies reassessed specifically for the possibility of the presence of an ectopic ACTH-secreting pititary adenoma that may not have been obvious on initial inspection.

Removal of a pituitary adenoma with preservation of pituitary function requires identification of the pseudocapsule of the tumor and selective separation of the tumor from the surrounding normal tissue. This pseudocapsule is formed from the compressed contiguous normal pituitary gland (specifically, the reticulin fibers) surrounding the expanding adenoma. In contrast, with tumors in the stalk, the delineation of the tumor edge is not as obvious as it is with tumors that arise from within the anterior lobe. In 
addition, the pial layer at the interface of the pseudocapsule of the tumor and the stalk is often quite tough, and sharp dissection is required to avoid excessive manipulation and preserve the anatomical integrity of the stalk. This goal was accomplished in all 10 patients in this study.

Transient hypocortisolism usually occurs after successful surgery for Cushing's disease.[18,27] Immediate postoperative hypocortisolism occurred in nine of the 10 patients and hypocortisolism occurred during the early postoperative interval in all patients. All patients required supplemental glucocorticoid therapy for a variable period (5-21 months). This variability of return of function to the hypothalamic-pituitary-adrenal axis is well known. $[8,10]$ The patient in Case 7 , whose adenoma originated in the stalk and who had eucortisolism immediately postoperatively and hypocortisolism 1 month after surgery, suffered recurrent Cushing's disease 31 months after surgery. Because most of the other patients have not been followed for as long as the patient in Case 7, Cushing's disease in other patients may also recur, although the incidence of recurrent Cushing's disease in patients with postoperative hypocortisolism is low.

The most frequent complication associated with transsphenoidal surgery is persistent CSF drainage. $[1,13]$ Other potential complications include oculomotor palsy, visual loss, diabetes insipidus, hypopituitarism, the empty sella syndrome with resulting prolapse of the optic chiasm, and death. Eight of our 10 patients had normal anterior pituitary function within 3 months. Three of our patients had preoperative hypothyroidism, which commonly occurs with Cushing's syndrome[5] and which usually disappears after resolution of hypercortisolism. $[8,15]$ Hypothyroidism resolved in two of these patients; the other patient had panhypopituitarism after surgery, which also occurred in the patient with preoperative hypogonadism and a tumor high in the pituitary stalk next to the optic chiasm. Three female patients with preoperative amenorrhea resumed normal menses after surgery. No patient had permanent diabetes insipidus (the patient in Case 10 is still following a course of DDAVP 5 months after surgery). One patient with preoperative hypogonadism underwent surgery only recently and the initial follow-up endocrinological assessment has not been performed.

One patient had bitemporal hemianopsia, primarily affecting the inferior temporal quadrants, immediately after surgery and has had no improvement during the early postoperative period. The loss of function of the portion of the optic chiasm distal from the tumor and the absence of recovery suggest that the bitemporal hemianopsia may have had a vascular cause, perhaps as a result of interruption of two small vessels that passed through the edge of the tumor to reach the chiasm. Although the chiasm receives its blood supply from multiple branches arising from the anterior cerebral and anterior communicating arteries superiorly and from branches of the internal carotid, superior hypophyseal, and posterior communicating arteries inferiorly,[2,14,22,23,25] most of the supply is from the superior surface, via branches of the anterior cerebral and anterior communicating arteries.[4,14,22,23,25] There is usually free intercommunication between the intrinsic capillary vessels of the optic chiasm and those of the hypothalamus, $[4,14,25]$ permitting excision of most tumors that arise from the stalk, such as craniopharyngiomas, without producing visual impairment. On the other hand, the largest, most numerous, and most extensive vessels in the plexus on the inferior surface of the optic chiasm are regularly supplied by the anterior superior hypophyseal arteries, $[4,14,25]$ which are distal branches of the superior hypophyseal arteries and which arise from the internal carotid artery and pass rostrally in the subarachnoid space to reach the pars tuberalis of the pituitary and inferior surface of the optic chiasm. The intrinsic capillary plexus of the chiasm, in turn, is connected to the vessels in the overlying pial network. Thus, occasionally postoperative loss of function in the optic chiasm may occur because of interruption of the superior hypophyseal arteries or their branches, which may have visual and endocrine 
consequences, as occurred in one of our patients.

\section{CONCLUSIONS}

Ten patients underwent transsphenoidal surgery for resection of ACTH-producing adenomas located in the pituitary stalk or extending into the stalk from the superior aspect of the anterior lobe. Previously, similar cases have been approached transcranially and most patients have lost pituitary function. Transsphenoidal exposure and selective adenomectomy permits clinical and laboratory remission of Cushing's disease with preservation of pituitary function in most patients.

\section{Acknowledgments}

We thank the medical artist, Mr. Howard Bartner, who is the Chief, Medical Illustration Section, Medical Arts and Photography Branch, National Center of Research Resources, National Institutes of Health, for so skillfully creating the drawings in Fig. 1. We also thank Prof. Wolfgang Seeger and Dr. Peter Warnke, Freiburg, Germany, for kindly referring the patient in Case 10 to our study.

\section{References}

1. Black PM, Zervas NT, Candia GL: Incidence and management of complications of transsphenoidal operation for pituitary adenomas. Neurosurgery 20:920-924, 1987

2. Cogan DG: Neurology of the Visual System. Springfield, Ill: Charles C Thomas, 1966

3. Colohan ART, Grady MS, Bonin JM, et al: Ectopic pituitary gland simulating a suprasellar tumor. Neurosurgery 20:43-48, 1987

4. Dawson BH: The blood vessels of the human optic chiasma and their relation to those of the hypophysis and hypothalamus. Brain 81:207-217, 1958

5. Duick DS, Wahner HW: Thyroid axis in patients with Cushing's syndrome. Arch Intern Med 139:767-772, 1979

6. Dyer EH, Civit T, Abecassis JP, et al: Functioning ectopic supradiaphragmatic pituitary adenomas. Neurosurgery 34:529-532, 1994

7. Dyer E H, Civit T, Visot A, et al: Transsphenoidal surgery for pituitary adenomas in children. Neurosurgery 34:207-212, 1994

8. Fitzgerald PA, Aron DC, Findling JW, et al: Cushing's disease: transient secondary adrenal insufficiency after selective removal of pituitary microadenomas; evidence for a pituitary origin. $\mathbf{J}$ Clin Endocrinol Metab 54:413-422, 1982

9. Flack MR, Oldfield EH, Cutler GB Jr, et al: Urine free cortisol in the high-dose dexamethasone suppression test for the differential diagnosis of the Cushing syndrome. Ann Intern Med 116:211-217, 1992

10. Graber AL, Ney RL, Nicholson WE, et al: Natural history of pituitary-adrenal recovery following long-term suppression with corticosteroids. J Clin Endocrinol 25:11-16, 1965

11. Hall WA, Luciano MG, Doppman JL, et al: Pituitary magnetic resonance imaging in normal human 
volunteers: occult adenomas in the general population. Ann Intern Med 120:817-820, 1994

12. Hamada J, Seto H, Miura M, et al: Suprasellar pituitary adenoma arising from the pars tuberalis: case report. Neurosurgery 27:647-649, 1990

13. Hardy J, McCutcheon IE: Pituitary microadenomas, in Apuzzo MLJ (ed): Brain Surgery. New York: Churchill Livingstone, 1993, pp 276-295

14. Hughes B: Blood supply of the optic nerves and chiasma and its clinical significance. Br $\mathbf{J}$ Ophthalmol 42:106-125, 1958

15. Kuwayama A, Kageyama N, Nakane T, et al: Anterior pituitary function after transsphenoidal selective adenomectomy in patients with Cushing's disease. J Clin Endocrinol Metab 53:165-173, 1981

16. Lafitte C, Aesch B, Henry-Lebras F, et al: Granular cell tumor of the pituitary stalk. Case report. J Neurosurg 80:1103-1107, 1994

17. Laws ER Jr: Transsphenoidal microsurgery in the management of craniopharyngioma. J Neurosurg 52:661-666, 1980

18. Magiakou MA, Mastorakos G, Oldfield EH, et al: Cushing's syndrome in children and adolescents. Presentation, diagnosis, and therapy. N Engl J Med 331:629-636, 1994

19. Matsumura A, Meguro K, Doi M, et al: Suprasellar ectopic pituitary adenoma: case report and review of the literature. Neurosurgery 26:681-685, 1990

20. Oldfield EH, Doppman JL, Nieman LK, et al: Petrosal sinus sampling with and without corticotropin-releasing hormone for the differential diagnosis of Cushing's syndrome. N Engl J Med 325:897-905, 1991

21. Ortiz-Suarez H, Erickson DL: Pituitary adenomas of adolescents. J Neurosurg 43:437-439, 1975

22. Renn WH, Rhoton AL Jr: Microsurgical anatomy of the sellar region. J Neurosurg 43:288-298, 1975

23. Rhoton AL Jr, Harris FS, Renn WH: Microsurgical anatomy of the sellar region and cavernous sinus, in Glaser JS (ed): Neuro-Ophthamology. St. Louis: CV Mosby, 1977, Vol 1, pp 75-105

24. Rothman LM, Sher J, Quencer RM, et al: Intracranial ectopic pituitary adenoma. J Neurosurg 44:96-99, 1976

25. Steele EJ, Blunt MJ: The blood supply of the optic nerve and chiasma in man. Case report. J Anat 90:486-493, 1956

26. Tal A: Cushing's disease caused by ectopic pituitary adenoma within the pituitary stalk. South Med J 86:249-250, 1993

27. Toms GC, McCarthy MI, Niven MJ, et al: Predicting relapse after transsphenoidal surgery for Cushing's disease. J Clin Endocrinol Metab 76:291-294, 1993

28. Wilson CB, Mindermann T, Tyrrell JB: Extrasellar, intracavernous sinus adrenocorticotropin-releasing adenoma causing Cushing's disease. J Clin Endocrinol Metab 
Manuscript received December 30, 1996.

Accepted in final form April 11, 1997.

Address reprint requests to: Edward H. Oldfield, M.D., Surgical Neurology Branch, National Institute of Neurological Disorders and Stroke, National Institutes of Health, Building 10, Room 5D-37, 9000 Rockville Pike, Bethesda, Maryland 20892. 